\title{
metal-organic compounds
}

Acta Crystallographica Section E

\section{Structure Reports}

Online

ISSN 1600-5368

\section{1-[(Z)-2-Cyano-2-(2-pyridyl)vinyl]- ferrocene}

\section{Xue-Qun Fu and Wei Wang*}

Ordered Matter Science Research Center, Southeast University, Nanjing 210096 , People's Republic of China

Correspondence e-mail: seuwangwei@gmail.com

Received 22 June 2008; accepted 14 July 2008

Key indicators: single-crystal X-ray study; $T=293 \mathrm{~K}$; mean $\sigma(\mathrm{C}-\mathrm{C})=0.004 \AA$;

$R$ factor $=0.042 ; w R$ factor $=0.094 ;$ data-to-parameter ratio $=17.4$.

In the title compound, $\left[\mathrm{Fe}\left(\mathrm{C}_{5} \mathrm{H}_{5}\right)\left(\mathrm{C}_{13} \mathrm{H}_{9} \mathrm{~N}_{2}\right)\right]$, the dihedral angle between the substituted cyclopentadienyl plane and the plane of the pyridine ring is $8.43(14)^{\circ}$. The double bond adopts a $Z$ configuration. In the crystal structure, weak $\mathrm{C}-$ $\mathrm{H} \cdots \mathrm{N}$ interactions link the molecules into a zigzag chain. A weak intramolecular $\mathrm{C}-\mathrm{H} \cdots \mathrm{N}$ hydrogen bond is also present.

\section{Related literature}

For the chemistry of ferrocene, see: Chen et al. (2006). For representative ferrocene derivatives, see: Jiao et al. (2003); Mancheno et al. (2004). For similar compounds, see: Boyd \& Paauwe (2006); Shao et al. (2005).

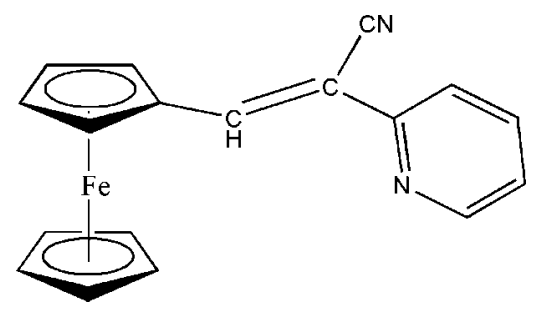

\section{Experimental}

Crystal data

$\left[\mathrm{Fe}\left(\mathrm{C}_{5} \mathrm{H}_{5}\right)\left(\mathrm{C}_{13} \mathrm{H}_{9} \mathrm{~N}_{2}\right)\right]$

$M_{r}=314.16$
Monoclinic, $P 2_{1} / n$

$a=11.105$ (2) А

$$
\begin{aligned}
& b=10.716(2) \AA \\
& c=12.675(3) \AA \\
& \beta=106.95(3)^{\circ} \\
& V=1442.9(5) \AA^{3} \\
& Z=4
\end{aligned}
$$

Data collection

Rigaku Mercury2 diffractometer Absorption correction: multi-scan (CrystalClear; Rigaku, 2005)

$T_{\min }=0.725, T_{\max }=0.900$

\section{Refinement}

$R\left[F^{2}>2 \sigma\left(F^{2}\right)\right]=0.041$

$w R\left(F^{2}\right)=0.094$

$S=1.07$

3311 reflections
Mo $K \alpha$ radiation

$\mu=1.04 \mathrm{~mm}^{-1}$

$T=293$ (2) K

$0.30 \times 0.20 \times 0.10 \mathrm{~mm}$

14799 measured reflections 3311 independent reflections 2564 reflections with $I>2 \sigma(I)$ $R_{\text {int }}=0.045$

190 parameters

$\mathrm{H}$-atom parameters constrained $\Delta \rho_{\max }=0.24{\mathrm{e} \AA^{-3}}^{-3}$

$\Delta \rho_{\min }=-0.24{\mathrm{e} \AA^{-3}}^{-3}$

Table 1

Hydrogen-bond geometry $\left(\AA{ }^{\circ}\right)$.

\begin{tabular}{lllll}
\hline$D-\mathrm{H} \cdots A$ & $D-\mathrm{H}$ & $\mathrm{H} \cdots A$ & $D \cdots A$ & $D-\mathrm{H} \cdots A$ \\
\hline $\mathrm{C} 16-\mathrm{H} 16 A \cdots \mathrm{N} 2$ & 0.93 & 2.41 & $2.804(3)$ & 105 \\
$\mathrm{C} 4-\mathrm{H} 4 A \cdots \mathrm{N} 1^{\mathrm{i}}$ & 0.98 & 2.62 & $3.538(4)$ & 156
\end{tabular}

Symmetry code: (i) $-x+1,-y+2,-z$.

Data collection: CrystalClear (Rigaku, 2005); cell refinement: CrystalClear; data reduction: CrystalClear; program(s) used to solve structure: SHELXS97 (Sheldrick, 2008); program(s) used to refine structure: SHELXL97 (Sheldrick, 2008); molecular graphics: SHELXTL (Sheldrick, 2008); software used to prepare material for publication: SHELXTL.

The authors are grateful to the Starter Fund of Southeast University for financial support to buy the CCD X-ray diffractometer.

Supplementary data and figures for this paper are available from the IUCr electronic archives (Reference: BQ2088).

\section{References}

Boyd, P. D. W. \& Paauwe, J. D. (2006). Acta Cryst. E62, m2153-m2155.

Chen, W., Mbafor, W., Roberts, S. M. \& Whittall, J. (2006). J. Am. Chem. Soc. 128, 3922-3923.

Jiao, J., Long, G. J., Grandjean, F., Beatty, A. M. \& Fehlner, T. P. (2003). J. Am. Chem. Soc. 125, 7522-7523.

Mancheno, O. G., Arrayas, R. G. \& Carretero, J. C. (2004). J. Am. Chem. Soc. 126, 456-457.

Rigaku (2005). CrystalClear. Rigaku Corporation, Tokyo, Japan.

Shao, L., Hu, Y., Tao, W.-F., Jin, Z. \& Fang, J.-X. (2005). Acta Cryst. E61, m1837-m1839.

Sheldrick, G. M. (2008). Acta Cryst. A64, 112-122. 


\section{supporting information}

Acta Cryst. (2008). E64, m1043 [doi:10.1107/S1600536808021843]

\section{1-[(Z)-2-Cyano-2-(2-pyridyl)vinyl]ferrocene}

\section{Xue-Qun Fu and Wei Wang}

\section{S1. Comment}

The molecular structure of the title compound is shown in Fig. 1. The Fe ${ }^{\cdots} C g 1$ and $\mathrm{Fe} \cdots C g 2$ distances are 1.6548 (16) $\AA$ and $1.6445(12) \AA$ respectively and the $C g 1 \cdots \mathrm{Fe} \cdots C g 2$ angle is $179.55(8)^{\circ}$, where $C g 1$ and $C g 2$ are the centroids of the unsubstituted and substituted cyclopentadienyl rings. The double bond $(\mathrm{C} 16=\mathrm{C} 17)$ exhibits a cis configuration and the pyridine plane makes an angle of 8.43 (14) $\AA$ with the substituted cyclopentadienyl ring. The planar cyclopentadienyl rings of the ferrocenyl unit are nearly parallel to each other [the interplanar angle is $1.33(17)^{\circ}$ ]. The crystal structure is stabilized by weak intramolecular $\mathrm{C}-\mathrm{H} \cdots \mathrm{N}$ interactions. Fig 2 shows that the molecules assemble as zigzag chains in the crystal structure along the $a$ axis, formed by weak intermolecular $\mathrm{C}-\mathrm{H} \cdots \mathrm{N}$ hydrogen bonds (Table 1).

\section{S2. Experimental}

$1 \mathrm{ml}$ pyrrolidine was added to the mixture of formylferrocene $(2.15 \mathrm{~g}, 0.01 \mathrm{~mol})$ and 2-pyridineacetonitrile $(1.18 \mathrm{~g}, 0.01$ $\mathrm{mol})$ in dichloromethane $(100 \mathrm{ml})$. The mixture was stirred at room temperature for $5 \mathrm{~h}$. After removing the solvent under reduced pressure, the residue was collected and dried in a vacuum desiccator. This crude product was purified by chromatography on silica gel, with petroleum ether and ethyl as eluant. Brownish red single crystals suitable for X-ray analysis were obtained by slow evaporation of ether at room temperature after several hours.

\section{S3. Refinement}

Positional parameters of all the $\mathrm{H}$ atoms were calculated geometrically and were allowed to ride on the $\mathrm{C}$ atoms to which they are bonded, with $U_{\text {iso }}(\mathrm{H})=1.2 U_{\text {eq }}(\mathrm{C})$. 


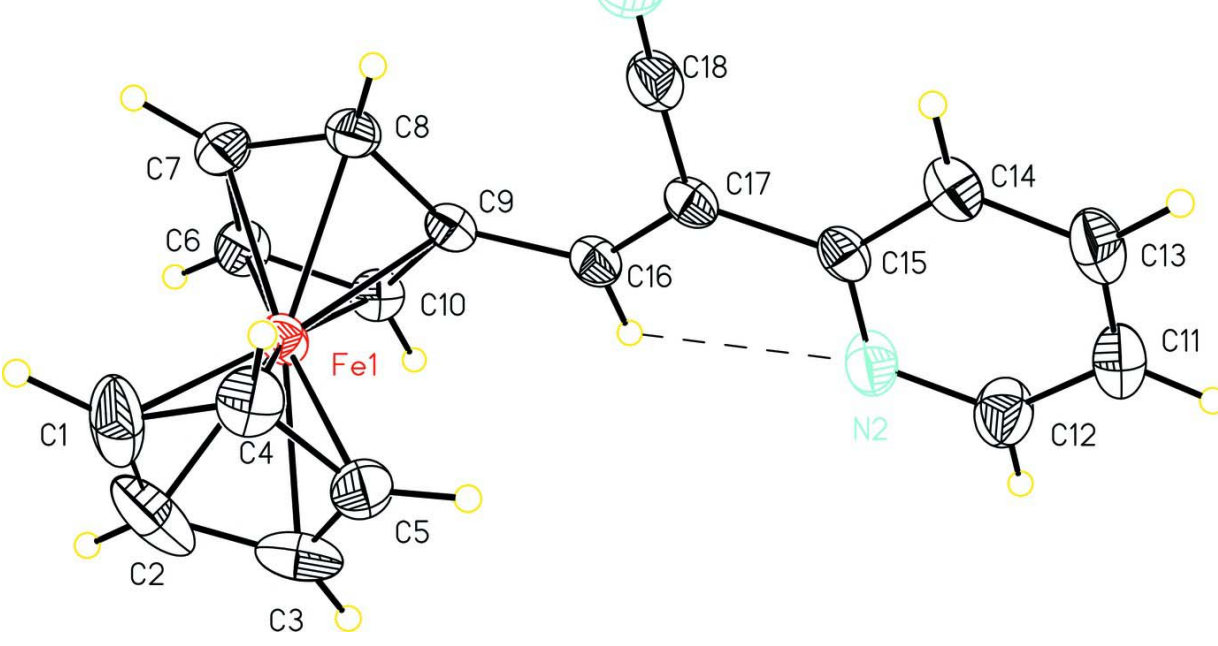

\section{Figure 1}

The molecular structure of the title compound, with displacement ellipsoids drawn at the $30 \%$ probability level. Hydrojen bond are shown as dashed lines 


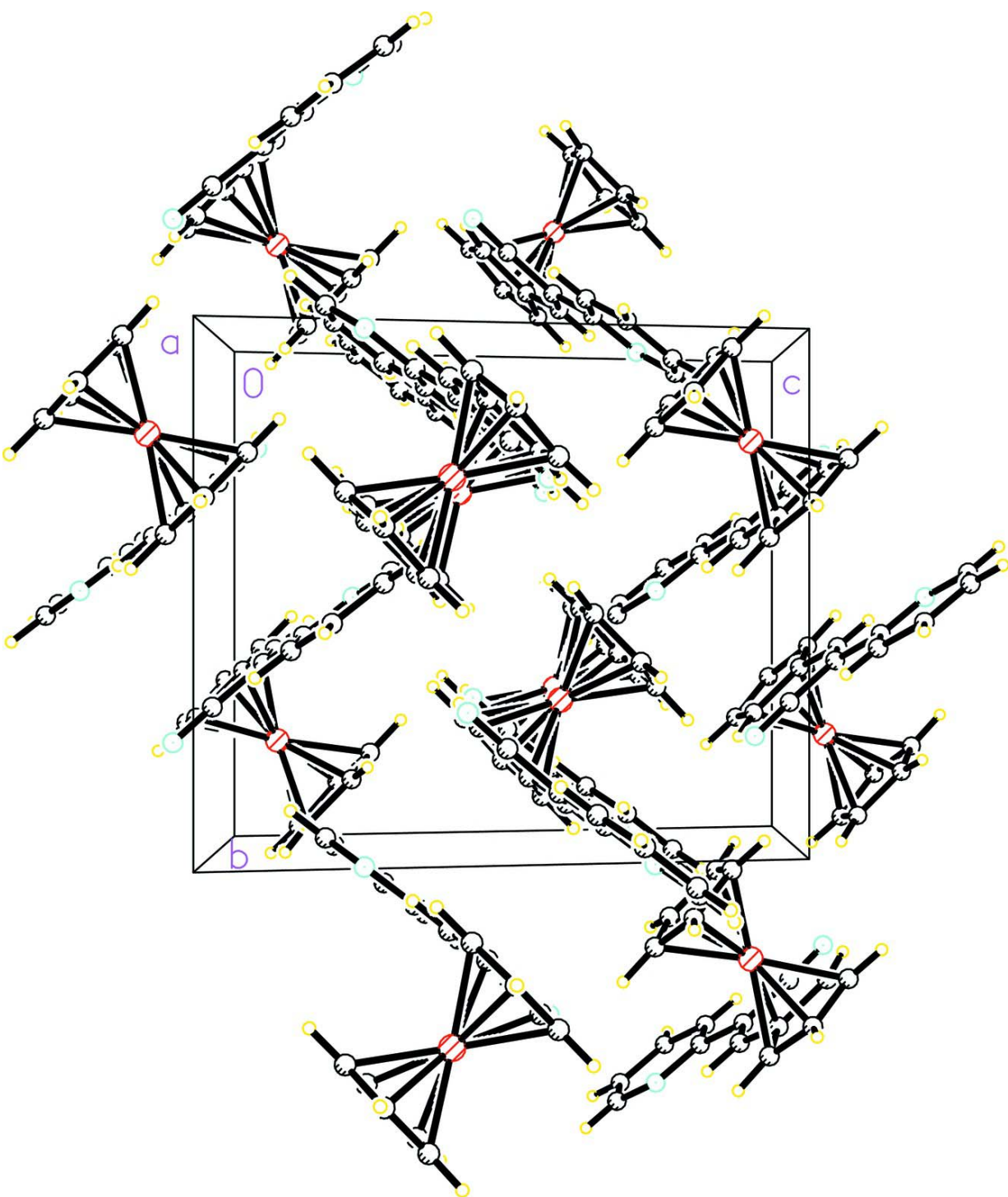

Figure 2

The packing diagram of the title compound, viewed along the $a$ axis.

\section{1-[(Z)-2-Cyano-2-(2-pyridyl)vinyl]ferrocene}

\section{Crystal data}

$\left[\mathrm{Fe}\left(\mathrm{C}_{5} \mathrm{H}_{5}\right)\left(\mathrm{C}_{13} \mathrm{H}_{9} \mathrm{~N}_{2}\right)\right]$

$M_{r}=314.16$

Monoclinic, $P 2{ }_{1} / n$

Hall symbol: -P $2 \mathrm{yn}$

$a=11.105(2) \AA$

$b=10.716(2) \AA$

$c=12.675(3) \AA$

$\beta=106.95(3)^{\circ}$

$V=1442.9(5) \AA^{3}$

$Z=4$
$F(000)=648$

$D_{\mathrm{x}}=1.446 \mathrm{Mg} \mathrm{m}^{-3}$

Mo $K \alpha$ radiation, $\lambda=0.71073 \AA$

Cell parameters from 12734 reflections

$\theta=6.7-55.3^{\circ}$

$\mu=1.04 \mathrm{~mm}^{-1}$

$T=293 \mathrm{~K}$

Prism, red brown

$0.30 \times 0.20 \times 0.10 \mathrm{~mm}$ 


\section{Data collection}

Rigaku Mercury2 (2x2 bin mode) diffractometer

Radiation source: fine-focus sealed tube Graphite monochromator

Detector resolution: 13.6612 pixels $\mathrm{mm}^{-1}$

CCD_Profile_fitting scans

Absorption correction: multi-scan

(CrystalClear; Rigaku, 2005)

$T_{\min }=0.725, T_{\max }=0.900$

Refinement

Refinement on $F^{2}$

Least-squares matrix: full

$R\left[F^{2}>2 \sigma\left(F^{2}\right)\right]=0.041$

$w R\left(F^{2}\right)=0.094$

$S=1.07$

3311 reflections

190 parameters

0 restraints

Primary atom site location: structure-invariant direct methods
14799 measured reflections

3311 independent reflections

2564 reflections with $I>2 \sigma(I)$

$R_{\text {int }}=0.045$

$\theta_{\max }=27.5^{\circ}, \theta_{\min }=3.4^{\circ}$

$h=-14 \rightarrow 14$

$k=-13 \rightarrow 13$

$l=-16 \rightarrow 16$
Secondary atom site location: difference Fourier map

Hydrogen site location: inferred from neighbouring sites

$\mathrm{H}$-atom parameters constrained

$w=1 /\left[\sigma^{2}\left(F_{\mathrm{o}}^{2}\right)+(0.0372 P)^{2}+0.4341 P\right]$

where $P=\left(F_{\mathrm{o}}^{2}+2 F_{\mathrm{c}}^{2}\right) / 3$

$(\Delta / \sigma)_{\max }<0.001$

$\Delta \rho_{\max }=0.24 \mathrm{e} \AA^{-3}$

$\Delta \rho_{\min }=-0.24$ e $\AA^{-3}$

\section{Special details}

Geometry. All e.s.d.'s (except the e.s.d. in the dihedral angle between two 1.s. planes) are estimated using the full covariance matrix. The cell e.s.d.'s are taken into account individually in the estimation of e.s.d.'s in distances, angles and torsion angles; correlations between e.s.d.'s in cell parameters are only used when they are defined by crystal symmetry. An approximate (isotropic) treatment of cell e.s.d.'s is used for estimating e.s.d.'s involving 1.s. planes.

Refinement. Refinement of $F^{2}$ against ALL reflections. The weighted $R$-factor $w R$ and goodness of fit $S$ are based on $F^{2}$, conventional $R$-factors $R$ are based on $F$, with $F$ set to zero for negative $F^{2}$. The threshold expression of $F^{2}>2 \sigma\left(F^{2}\right)$ is used only for calculating $R$-factors $(\mathrm{gt})$ etc. and is not relevant to the choice of reflections for refinement. $R$-factors based on $F^{2}$ are statistically about twice as large as those based on $F$, and $R$-factors based on ALL data will be even larger.

Fractional atomic coordinates and isotropic or equivalent isotropic displacement parameters $\left(\AA^{2}\right)$

\begin{tabular}{lllll}
\hline & $x$ & $y$ & $z$ & $U_{\text {iso }} * U_{\text {eq }}$ \\
\hline Fe1 & $0.19959(3)$ & $0.79543(3)$ & $0.08768(3)$ & $0.03864(12)$ \\
C10 & $0.1744(2)$ & $0.6098(2)$ & $0.0568(2)$ & $0.0452(5)$ \\
H10A & 0.1703 & 0.5456 & 0.1108 & $0.054^{*}$ \\
C9 & $0.2868(2)$ & $0.6523(2)$ & $0.03368(18)$ & $0.0400(5)$ \\
C15 & $0.6436(2)$ & $0.5798(2)$ & $0.15481(19)$ & $0.0422(5)$ \\
C17 & $0.5244(2)$ & $0.6339(2)$ & $0.08206(18)$ & $0.0404(5)$ \\
C16 & $0.4110(2)$ & $0.6083(2)$ & $0.09516(19)$ & $0.0419(5)$ \\
H16A & 0.4124 & 0.5539 & 0.1526 & $0.050^{*}$ \\
N2 & $0.63206(19)$ & $0.5064(2)$ & $0.23633(19)$ & $0.0559(6)$ \\
C18 & $0.5387(2)$ & $0.7169(3)$ & $-0.0022(2)$ & $0.0523(6)$ \\
C8 & $0.2495(2)$ & $0.7467(2)$ & $-0.04956(19)$ & $0.0444(5)$ \\
H8A & 0.3056 & 0.7937 & -0.0820 & $0.053 *$ \\
C7 & $0.1167(2)$ & $0.7601(3)$ & $-0.0764(2)$ & $0.0489(6)$ \\
H7A & 0.0656 & 0.8192 & -0.1301 & $0.059^{*}$ \\
N1 & $0.5562(3)$ & $0.7837(3)$ & $-0.0659(2)$ & $0.0831(9)$ \\
C14 & $0.7600(2)$ & $0.6058(3)$ & $0.1397(2)$ & $0.0545(6)$
\end{tabular}




$\begin{array}{lllll}\text { H1A } & 0.7658 & 0.6568 & 0.0820 & 0.065^{*} \\ \text { C5 } & 0.3262(2) & 0.8697(3) & 0.2228(2) & 0.0551(7) \\ \text { H5A } & 0.4138 & 0.8427 & 0.2532 & 0.066^{*} \\ \text { C11 } & 0.8554(3) & 0.4798(3) & 0.2947(3) & 0.0659(8) \\ \text { H11A } & 0.9260 & 0.4443 & 0.3441 & 0.079^{*} \\ \text { C13 } & 0.8671(2) & 0.5547(3) & 0.2116(3) & 0.0658(8) \\ \text { H13A } & 0.9460 & 0.5714 & 0.2032 & 0.079^{*} \\ \text { C4 } & 0.2841(3) & 0.9625(2) & 0.1432(2) & 0.0570(7) \\ \text { H4A } & 0.3362 & 1.0123 & 0.1086 & 0.068^{*} \\ \text { C6 } & 0.0708(2) & 0.6760(2) & -0.0113(2) & 0.0494(6) \\ \text { H6A } & -0.0172 & 0.6664 & -0.0122 & 0.059^{*} \\ \text { C2 } & 0.1158(3) & 0.8840(4) & 0.1894(4) & 0.0946(13) \\ \text { H2A } & 0.0298 & 0.8700 & 0.1927 & 0.114^{*} \\ \text { C12 } & 0.7372(3) & 0.4577(3) & 0.3038(3) & 0.0667(8) \\ \text { H12A } & 0.7298 & 0.4058 & 0.3604 & 0.080^{*} \\ \text { C3 } & 0.2238(4) & 0.8212(3) & 0.2518(2) & 0.0751(10) \\ \text { H3A } & 0.2268 & 0.7556 & 0.3063 & 0.090^{*} \\ \text { C1 } & 0.1528(3) & 0.9719(3) & 0.1227(3) & 0.0840(11) \\ \text { H1B } & 0.0969 & 1.0294 & 0.0707 & 0.101^{*} \\ \end{array}$

Atomic displacement parameters $\left(\AA^{2}\right)$

\begin{tabular}{lllllll}
\hline & $U^{11}$ & $U^{22}$ & $U^{33}$ & $U^{12}$ & $U^{13}$ & $U^{23}$ \\
\hline Fe1 & $0.03100(18)$ & $0.0435(2)$ & $0.0417(2)$ & $-0.00034(14)$ & $0.01115(13)$ & $-0.00617(15)$ \\
C10 & $0.0451(13)$ & $0.0435(13)$ & $0.0463(13)$ & $-0.0074(10)$ & $0.0122(10)$ & $-0.0034(11)$ \\
C9 & $0.0417(12)$ & $0.0401(12)$ & $0.0412(12)$ & $-0.0010(10)$ & $0.0167(10)$ & $-0.0059(10)$ \\
C15 & $0.0434(13)$ & $0.0364(12)$ & $0.0505(14)$ & $0.0016(10)$ & $0.0195(11)$ & $-0.0089(10)$ \\
C17 & $0.0461(13)$ & $0.0351(12)$ & $0.0445(13)$ & $0.0016(10)$ & $0.0204(10)$ & $-0.0062(10)$ \\
C16 & $0.0459(13)$ & $0.0381(12)$ & $0.0454(13)$ & $0.0026(10)$ & $0.0193(10)$ & $-0.0023(10)$ \\
N2 & $0.0445(12)$ & $0.0576(13)$ & $0.0678(14)$ & $0.0062(10)$ & $0.0198(11)$ & $0.0123(11)$ \\
C18 & $0.0431(14)$ & $0.0598(17)$ & $0.0593(16)$ & $0.0034(12)$ & $0.0233(12)$ & $0.0040(13)$ \\
C8 & $0.0453(13)$ & $0.0511(13)$ & $0.0397(12)$ & $0.0000(11)$ & $0.0170(11)$ & $-0.0011(11)$ \\
C7 & $0.0461(14)$ & $0.0572(15)$ & $0.0388(13)$ & $0.0002(11)$ & $0.0051(11)$ & $-0.0016(11)$ \\
N1 & $0.0676(17)$ & $0.100(2)$ & $0.090(2)$ & $0.0035(15)$ & $0.0367(15)$ & $0.0364(17)$ \\
C14 & $0.0470(14)$ & $0.0577(16)$ & $0.0645(17)$ & $0.0010(12)$ & $0.0255(13)$ & $-0.0014(13)$ \\
C5 & $0.0505(14)$ & $0.0611(17)$ & $0.0459(14)$ & $0.0000(13)$ & $0.0016(12)$ & $-0.0139(13)$ \\
C11 & $0.0457(15)$ & $0.0678(19)$ & $0.081(2)$ & $0.0107(14)$ & $0.0126(14)$ & $0.0006(16)$ \\
C13 & $0.0404(14)$ & $0.075(2)$ & $0.085(2)$ & $0.0026(14)$ & $0.0225(14)$ & $-0.0074(17)$ \\
C4 & $0.0566(16)$ & $0.0460(15)$ & $0.0618(17)$ & $-0.0087(12)$ & $0.0072(13)$ & $-0.0111(13)$ \\
C6 & $0.0359(12)$ & $0.0571(16)$ & $0.0512(14)$ & $-0.0097(11)$ & $0.0066(11)$ & $-0.0082(12)$ \\
C2 & $0.058(2)$ & $0.114(3)$ & $0.126(3)$ & $-0.019(2)$ & $0.050(2)$ & $-0.073(3)$ \\
C12 & $0.0573(17)$ & $0.068(2)$ & $0.0741(19)$ & $0.0057(14)$ & $0.0184(15)$ & $0.0180(15)$ \\
C3 & $0.107(3)$ & $0.079(2)$ & $0.0500(17)$ & $-0.017(2)$ & $0.0393(18)$ & $-0.0222(15)$ \\
C1 & $0.064(2)$ & $0.061(2)$ & $0.106(3)$ & $0.0237(16)$ & $-0.0094(19)$ & $-0.0369(19)$ \\
& & & & & &
\end{tabular}


Geometric parameters $\left(\AA,{ }^{\circ}\right)$

\begin{tabular}{|c|c|c|c|}
\hline $\mathrm{Fe} 1-\mathrm{C} 10$ & $2.031(2)$ & $\mathrm{C} 8-\mathrm{C} 7$ & $1.421(3)$ \\
\hline $\mathrm{Fe} 1-\mathrm{C} 2$ & $2.032(3)$ & $\mathrm{C} 8-\mathrm{H} 8 \mathrm{~A}$ & 0.9800 \\
\hline $\mathrm{Fe} 1-\mathrm{C} 5$ & $2.034(2)$ & $\mathrm{C} 7-\mathrm{C} 6$ & $1.414(4)$ \\
\hline $\mathrm{Fe} 1-\mathrm{C} 9$ & $2.036(2)$ & C7-H7A & 0.9800 \\
\hline $\mathrm{Fe} 1-\mathrm{C} 3$ & $2.037(3)$ & $\mathrm{C} 14-\mathrm{C} 13$ & $1.383(4)$ \\
\hline $\mathrm{Fe} 1-\mathrm{C} 8$ & $2.042(2)$ & $\mathrm{C} 14-\mathrm{H} 1 \mathrm{~A}$ & 0.9300 \\
\hline $\mathrm{Fe} 1-\mathrm{C} 4$ & $2.048(3)$ & $\mathrm{C} 5-\mathrm{C} 3$ & $1.393(4)$ \\
\hline $\mathrm{Fe} 1-\mathrm{C} 7$ & $2.050(2)$ & $\mathrm{C} 5-\mathrm{C} 4$ & $1.397(4)$ \\
\hline $\mathrm{Fe} 1-\mathrm{C} 6$ & $2.052(2)$ & $\mathrm{C} 5-\mathrm{H} 5 \mathrm{~A}$ & 0.9800 \\
\hline $\mathrm{Fe} 1-\mathrm{C} 1$ & $2.044(3)$ & $\mathrm{C} 11-\mathrm{C} 13$ & $1.361(4)$ \\
\hline $\mathrm{C} 10-\mathrm{C} 6$ & $1.411(3)$ & $\mathrm{C} 11-\mathrm{C} 12$ & $1.372(4)$ \\
\hline $\mathrm{C} 10-\mathrm{C} 9$ & $1.438(3)$ & $\mathrm{C} 11-\mathrm{H} 11 \mathrm{~A}$ & 0.9300 \\
\hline $\mathrm{C} 10-\mathrm{H} 10 \mathrm{~A}$ & 0.9800 & $\mathrm{C} 13-\mathrm{H} 13 \mathrm{~A}$ & 0.9300 \\
\hline $\mathrm{C} 9-\mathrm{C} 8$ & $1.432(3)$ & $\mathrm{C} 4-\mathrm{C} 1$ & $1.408(4)$ \\
\hline $\mathrm{C} 9-\mathrm{C} 16$ & $1.450(3)$ & $\mathrm{C} 4-\mathrm{H} 4 \mathrm{~A}$ & 0.9800 \\
\hline $\mathrm{C} 15-\mathrm{N} 2$ & $1.334(3)$ & $\mathrm{C} 6-\mathrm{H} 6 \mathrm{~A}$ & 0.9800 \\
\hline $\mathrm{C} 15-\mathrm{C} 14$ & $1.389(3)$ & $\mathrm{C} 2-\mathrm{C} 1$ & $1.404(5)$ \\
\hline $\mathrm{C} 15-\mathrm{C} 17$ & $1.492(3)$ & $\mathrm{C} 2-\mathrm{C} 3$ & $1.401(5)$ \\
\hline $\mathrm{C} 17-\mathrm{C} 16$ & $1.346(3)$ & $\mathrm{C} 2-\mathrm{H} 2 \mathrm{~A}$ & 0.9800 \\
\hline $\mathrm{C} 17-\mathrm{C} 18$ & $1.433(3)$ & $\mathrm{C} 12-\mathrm{H} 12 \mathrm{~A}$ & 0.9300 \\
\hline $\mathrm{C} 16-\mathrm{H} 16 \mathrm{~A}$ & 0.9300 & $\mathrm{C} 3-\mathrm{H} 3 \mathrm{~A}$ & 0.9800 \\
\hline $\mathrm{N} 2-\mathrm{C} 12$ & $1.336(3)$ & $\mathrm{C} 1-\mathrm{H} 1 \mathrm{~B}$ & 0.9800 \\
\hline $\mathrm{C} 18-\mathrm{N} 1$ & $1.138(3)$ & & \\
\hline $\mathrm{C} 10-\mathrm{Fe} 1-\mathrm{C} 2$ & $121.13(14)$ & $\mathrm{C} 17-\mathrm{C} 16-\mathrm{H} 16 \mathrm{~A}$ & 114.9 \\
\hline $\mathrm{C} 10-\mathrm{Fe} 1-\mathrm{C} 5$ & $124.61(11)$ & $\mathrm{C} 9-\mathrm{C} 16-\mathrm{H} 16 \mathrm{~A}$ & 114.9 \\
\hline $\mathrm{C} 2-\mathrm{Fe} 1-\mathrm{C} 5$ & $67.36(12)$ & $\mathrm{C} 15-\mathrm{N} 2-\mathrm{C} 12$ & $117.6(2)$ \\
\hline $\mathrm{C} 10-\mathrm{Fe} 1-\mathrm{C} 9$ & $41.40(9)$ & $\mathrm{N} 1-\mathrm{C} 18-\mathrm{C} 17$ & $176.5(3)$ \\
\hline $\mathrm{C} 2-\mathrm{Fe} 1-\mathrm{C} 9$ & $156.35(17)$ & $\mathrm{C} 7-\mathrm{C} 8-\mathrm{C} 9$ & $107.8(2)$ \\
\hline $\mathrm{C} 5-\mathrm{Fe} 1-\mathrm{C} 9$ & $107.32(10)$ & $\mathrm{C} 7-\mathrm{C} 8-\mathrm{Fe} 1$ & $69.98(14)$ \\
\hline $\mathrm{C} 10-\mathrm{Fe} 1-\mathrm{C} 3$ & $107.43(12)$ & $\mathrm{C} 9-\mathrm{C} 8-\mathrm{Fe} 1$ & $69.22(13)$ \\
\hline $\mathrm{C} 2-\mathrm{Fe} 1-\mathrm{C} 3$ & $40.27(14)$ & $\mathrm{C} 7-\mathrm{C} 8-\mathrm{H} 8 \mathrm{~A}$ & 126.1 \\
\hline $\mathrm{C} 5-\mathrm{Fe} 1-\mathrm{C} 3$ & $40.03(12)$ & $\mathrm{C} 9-\mathrm{C} 8-\mathrm{H} 8 \mathrm{~A}$ & 126.1 \\
\hline $\mathrm{C} 9-\mathrm{Fe} 1-\mathrm{C} 3$ & $120.76(13)$ & $\mathrm{Fe} 1-\mathrm{C} 8-\mathrm{H} 8 \mathrm{~A}$ & 126.1 \\
\hline $\mathrm{C} 10-\mathrm{Fe} 1-\mathrm{C} 8$ & $68.99(10)$ & $\mathrm{C} 6-\mathrm{C} 7-\mathrm{C} 8$ & $108.7(2)$ \\
\hline $\mathrm{C} 2-\mathrm{Fe} 1-\mathrm{C} 8$ & $161.54(16)$ & $\mathrm{C} 6-\mathrm{C} 7-\mathrm{Fe} 1$ & $69.92(14)$ \\
\hline $\mathrm{C} 5-\mathrm{Fe} 1-\mathrm{C} 8$ & $121.64(11)$ & $\mathrm{C} 8-\mathrm{C} 7-\mathrm{Fe} 1$ & $69.38(14)$ \\
\hline $\mathrm{C} 9-\mathrm{Fe} 1-\mathrm{C} 8$ & $41.13(9)$ & $\mathrm{C} 6-\mathrm{C} 7-\mathrm{H} 7 \mathrm{~A}$ & 125.6 \\
\hline $\mathrm{C} 3-\mathrm{Fe} 1-\mathrm{C} 8$ & $156.49(13)$ & $\mathrm{C} 8-\mathrm{C} 7-\mathrm{H} 7 \mathrm{~A}$ & 125.6 \\
\hline $\mathrm{C} 10-\mathrm{Fe} 1-\mathrm{C} 4$ & $161.07(10)$ & $\mathrm{Fe} 1-\mathrm{C} 7-\mathrm{H} 7 \mathrm{~A}$ & 125.6 \\
\hline $\mathrm{C} 2-\mathrm{Fe} 1-\mathrm{C} 4$ & $67.80(14)$ & $\mathrm{C} 13-\mathrm{C} 14-\mathrm{C} 15$ & $119.1(3)$ \\
\hline $\mathrm{C} 5-\mathrm{Fe} 1-\mathrm{C} 4$ & $40.01(11)$ & $\mathrm{C} 13-\mathrm{C} 14-\mathrm{H} 1 \mathrm{~A}$ & 120.5 \\
\hline $\mathrm{C} 9-\mathrm{Fe} 1-\mathrm{C} 4$ & $123.96(10)$ & $\mathrm{C} 15-\mathrm{C} 14-\mathrm{H} 1 \mathrm{~A}$ & 120.5 \\
\hline $\mathrm{C} 3-\mathrm{Fe} 1-\mathrm{C} 4$ & $67.64(12)$ & $\mathrm{C} 3-\mathrm{C} 5-\mathrm{C} 4$ & $109.2(3)$ \\
\hline $\mathrm{C} 8-\mathrm{Fe} 1-\mathrm{C} 4$ & $107.79(11)$ & $\mathrm{C} 3-\mathrm{C} 5-\mathrm{Fe} 1$ & $70.08(16)$ \\
\hline $\mathrm{C} 10-\mathrm{Fe} 1-\mathrm{C} 7$ & $68.15(10)$ & $\mathrm{C} 4-\mathrm{C} 5-\mathrm{Fe} 1$ & $70.54(14)$ \\
\hline
\end{tabular}




\begin{tabular}{|c|c|c|c|}
\hline $\mathrm{C} 2-\mathrm{Fe} 1-\mathrm{C} 7$ & $125.25(14)$ & $\mathrm{C} 3-\mathrm{C} 5-\mathrm{H} 5 \mathrm{~A}$ & 125.4 \\
\hline $\mathrm{C} 5-\mathrm{Fe} 1-\mathrm{C} 7$ & $157.24(11)$ & $\mathrm{C} 4-\mathrm{C} 5-\mathrm{H} 5 \mathrm{~A}$ & 125.4 \\
\hline $\mathrm{C} 9-\mathrm{Fe} 1-\mathrm{C} 7$ & $68.70(10)$ & $\mathrm{Fe} 1-\mathrm{C} 5-\mathrm{H} 5 \mathrm{~A}$ & 125.4 \\
\hline $\mathrm{C} 3-\mathrm{Fe} 1-\mathrm{C} 7$ & $161.43(13)$ & $\mathrm{C} 13-\mathrm{C} 11-\mathrm{C} 12$ & $118.5(3)$ \\
\hline $\mathrm{C} 8-\mathrm{Fe} 1-\mathrm{C} 7$ & $40.64(9)$ & $\mathrm{C} 13-\mathrm{C} 11-\mathrm{H} 11 \mathrm{~A}$ & 120.8 \\
\hline $\mathrm{C} 4-\mathrm{Fe} 1-\mathrm{C} 7$ & $122.35(11)$ & $\mathrm{C} 12-\mathrm{C} 11-\mathrm{H} 11 \mathrm{~A}$ & 120.8 \\
\hline $\mathrm{C} 10-\mathrm{Fe} 1-\mathrm{C} 6$ & $40.43(9)$ & $\mathrm{C} 11-\mathrm{C} 13-\mathrm{C} 14$ & $119.1(3)$ \\
\hline $\mathrm{C} 2-\mathrm{Fe} 1-\mathrm{C} 6$ & $108.25(12)$ & $\mathrm{C} 11-\mathrm{C} 13-\mathrm{H} 13 \mathrm{~A}$ & 120.5 \\
\hline $\mathrm{C} 5-\mathrm{Fe} 1-\mathrm{C} 6$ & $161.08(11)$ & $\mathrm{C} 14-\mathrm{C} 13-\mathrm{H} 13 \mathrm{~A}$ & 120.5 \\
\hline $\mathrm{C} 9-\mathrm{Fe} 1-\mathrm{C} 6$ & $68.88(10)$ & $\mathrm{C} 5-\mathrm{C} 4-\mathrm{C} 1$ & $107.2(3)$ \\
\hline $\mathrm{C} 3-\mathrm{Fe} 1-\mathrm{C} 6$ & $124.75(12)$ & $\mathrm{C} 5-\mathrm{C} 4-\mathrm{Fe} 1$ & $69.45(15)$ \\
\hline $\mathrm{C} 8-\mathrm{Fe} 1-\mathrm{C} 6$ & $68.49(10)$ & $\mathrm{C} 1-\mathrm{C} 4-\mathrm{Fe} 1$ & $69.68(16)$ \\
\hline $\mathrm{C} 4-\mathrm{Fe} 1-\mathrm{C} 6$ & $157.37(11)$ & $\mathrm{C} 5-\mathrm{C} 4-\mathrm{H} 4 \mathrm{~A}$ & 126.4 \\
\hline $\mathrm{C} 7-\mathrm{Fe} 1-\mathrm{C} 6$ & $40.34(10)$ & $\mathrm{C} 1-\mathrm{C} 4-\mathrm{H} 4 \mathrm{~A}$ & 126.4 \\
\hline $\mathrm{C} 10-\mathrm{Fe} 1-\mathrm{C} 1$ & $156.70(13)$ & $\mathrm{Fe} 1-\mathrm{C} 4-\mathrm{H} 4 \mathrm{~A}$ & 126.4 \\
\hline $\mathrm{C} 2-\mathrm{Fe} 1-\mathrm{C} 1$ & $40.31(16)$ & $\mathrm{C} 10-\mathrm{C} 6-\mathrm{C} 7$ & $108.0(2)$ \\
\hline $\mathrm{C} 5-\mathrm{Fe} 1-\mathrm{C} 1$ & $67.21(12)$ & $\mathrm{C} 10-\mathrm{C} 6-\mathrm{Fe} 1$ & $68.98(13)$ \\
\hline $\mathrm{C} 9-\mathrm{Fe} 1-\mathrm{C} 1$ & $161.07(15)$ & $\mathrm{C} 7-\mathrm{C} 6-\mathrm{Fe} 1$ & $69.74(14)$ \\
\hline $\mathrm{C} 3-\mathrm{Fe} 1-\mathrm{C} 1$ & $67.60(15)$ & $\mathrm{C} 10-\mathrm{C} 6-\mathrm{H} 6 \mathrm{~A}$ & 126.0 \\
\hline $\mathrm{C} 8-\mathrm{Fe} 1-\mathrm{C} 1$ & $124.84(15)$ & $\mathrm{C} 7-\mathrm{C} 6-\mathrm{H} 6 \mathrm{~A}$ & 126.0 \\
\hline $\mathrm{C} 4-\mathrm{Fe} 1-\mathrm{C} 1$ & $40.26(12)$ & $\mathrm{Fe} 1-\mathrm{C} 6-\mathrm{H} 6 \mathrm{~A}$ & 126.0 \\
\hline $\mathrm{C} 7-\mathrm{Fe} 1-\mathrm{C} 1$ & $108.88(13)$ & $\mathrm{C} 1-\mathrm{C} 2-\mathrm{C} 3$ & $108.0(3)$ \\
\hline $\mathrm{C} 6-\mathrm{Fe} 1-\mathrm{C} 1$ & $122.26(12)$ & $\mathrm{C} 1-\mathrm{C} 2-\mathrm{Fe} 1$ & $70.29(18)$ \\
\hline $\mathrm{C} 6-\mathrm{C} 10-\mathrm{C} 9$ & $108.5(2)$ & $\mathrm{C} 3-\mathrm{C} 2-\mathrm{Fe} 1$ & $70.05(17)$ \\
\hline $\mathrm{C} 6-\mathrm{C} 10-\mathrm{Fe} 1$ & $70.59(14)$ & $\mathrm{C} 1-\mathrm{C} 2-\mathrm{H} 2 \mathrm{~A}$ & 126.0 \\
\hline $\mathrm{C} 9-\mathrm{C} 10-\mathrm{Fe} 1$ & $69.48(13)$ & $\mathrm{C} 3-\mathrm{C} 2-\mathrm{H} 2 \mathrm{~A}$ & 126.0 \\
\hline $\mathrm{C} 6-\mathrm{C} 10-\mathrm{H} 10 \mathrm{~A}$ & 125.7 & $\mathrm{Fe} 1-\mathrm{C} 2-\mathrm{H} 2 \mathrm{~A}$ & 126.0 \\
\hline $\mathrm{C} 9-\mathrm{C} 10-\mathrm{H} 10 \mathrm{~A}$ & 125.7 & $\mathrm{~N} 2-\mathrm{C} 12-\mathrm{C} 11$ & $123.9(3)$ \\
\hline $\mathrm{Fe} 1-\mathrm{C} 10-\mathrm{H} 10 \mathrm{~A}$ & 125.7 & $\mathrm{~N} 2-\mathrm{C} 12-\mathrm{H} 12 \mathrm{~A}$ & 118.0 \\
\hline $\mathrm{C} 8-\mathrm{C} 9-\mathrm{C} 10$ & $107.0(2)$ & $\mathrm{C} 11-\mathrm{C} 12-\mathrm{H} 12 \mathrm{~A}$ & 118.0 \\
\hline $\mathrm{C} 8-\mathrm{C} 9-\mathrm{C} 16$ & $130.6(2)$ & $\mathrm{C} 5-\mathrm{C} 3-\mathrm{C} 2$ & $107.6(3)$ \\
\hline $\mathrm{C} 10-\mathrm{C} 9-\mathrm{C} 16$ & $122.2(2)$ & $\mathrm{C} 5-\mathrm{C} 3-\mathrm{Fe} 1$ & $69.89(16)$ \\
\hline $\mathrm{C} 8-\mathrm{C} 9-\mathrm{Fe} 1$ & $69.65(13)$ & $\mathrm{C} 2-\mathrm{C} 3-\mathrm{Fe} 1$ & $69.68(19)$ \\
\hline $\mathrm{C} 10-\mathrm{C} 9-\mathrm{Fe} 1$ & $69.11(13)$ & $\mathrm{C} 5-\mathrm{C} 3-\mathrm{H} 3 \mathrm{~A}$ & 126.2 \\
\hline $\mathrm{C} 16-\mathrm{C} 9-\mathrm{Fe} 1$ & $122.35(16)$ & $\mathrm{C} 2-\mathrm{C} 3-\mathrm{H} 3 \mathrm{~A}$ & 126.2 \\
\hline $\mathrm{N} 2-\mathrm{C} 15-\mathrm{C} 14$ & $121.9(2)$ & $\mathrm{Fe} 1-\mathrm{C} 3-\mathrm{H} 3 \mathrm{~A}$ & 126.2 \\
\hline $\mathrm{N} 2-\mathrm{C} 15-\mathrm{C} 17$ & $116.1(2)$ & $\mathrm{C} 2-\mathrm{C} 1-\mathrm{C} 4$ & $108.0(3)$ \\
\hline $\mathrm{C} 14-\mathrm{C} 15-\mathrm{C} 17$ & $122.0(2)$ & $\mathrm{C} 2-\mathrm{C} 1-\mathrm{Fe} 1$ & $69.40(18)$ \\
\hline $\mathrm{C} 16-\mathrm{C} 17-\mathrm{C} 18$ & $122.0(2)$ & $\mathrm{C} 4-\mathrm{C} 1-\mathrm{Fe} 1$ & $70.06(15)$ \\
\hline $\mathrm{C} 16-\mathrm{C} 17-\mathrm{C} 15$ & $122.5(2)$ & $\mathrm{C} 2-\mathrm{C} 1-\mathrm{H} 1 \mathrm{~B}$ & 126.0 \\
\hline $\mathrm{C} 18-\mathrm{C} 17-\mathrm{C} 15$ & $115.5(2)$ & $\mathrm{C} 4-\mathrm{C} 1-\mathrm{H} 1 \mathrm{~B}$ & 126.0 \\
\hline $\mathrm{C} 17-\mathrm{C} 16-\mathrm{C} 9$ & $130.1(2)$ & $\mathrm{Fe} 1-\mathrm{C} 1-\mathrm{H} 1 \mathrm{~B}$ & 126.0 \\
\hline $\mathrm{C} 2-\mathrm{Fe} 1-\mathrm{C} 10-\mathrm{C} 6$ & $81.7(2)$ & $\mathrm{C} 3-\mathrm{Fe} 1-\mathrm{C} 5-\mathrm{C} 4$ & $-119.9(3)$ \\
\hline $\mathrm{C} 5-\mathrm{Fe} 1-\mathrm{C} 10-\mathrm{C} 6$ & $164.22(16)$ & $\mathrm{C} 8-\mathrm{Fe} 1-\mathrm{C} 5-\mathrm{C} 4$ & $79.87(19)$ \\
\hline $\mathrm{C} 9-\mathrm{Fe} 1-\mathrm{C} 10-\mathrm{C} 6$ & $-119.4(2)$ & $\mathrm{C} 7-\mathrm{Fe} 1-\mathrm{C} 5-\mathrm{C} 4$ & $46.5(4)$ \\
\hline $\mathrm{C} 3-\mathrm{Fe} 1-\mathrm{C} 10-\mathrm{C} 6$ & $123.55(18)$ & $\mathrm{C} 6-\mathrm{Fe} 1-\mathrm{C} 5-\mathrm{C} 4$ & $-162.1(3)$ \\
\hline $\mathrm{C} 8-\mathrm{Fe} 1-\mathrm{C} 10-\mathrm{C} 6$ & $-81.09(16)$ & $\mathrm{C} 1-\mathrm{Fe} 1-\mathrm{C} 5-\mathrm{C} 4$ & $-38.1(2)$ \\
\hline
\end{tabular}




\begin{tabular}{|c|c|}
\hline $\mathrm{C} 4-\mathrm{Fe} 1-\mathrm{C} 10-\mathrm{C} 6$ & $-164.7(3)$ \\
\hline $\mathrm{C} 7-\mathrm{Fe} 1-\mathrm{C} 10-\mathrm{C} 6$ & $-37.29(15)$ \\
\hline $\mathrm{C} 1-\mathrm{Fe} 1-\mathrm{C} 10-\mathrm{C} 6$ & $49.9(4)$ \\
\hline $\mathrm{C} 2-\mathrm{Fe} 1-\mathrm{C} 10-\mathrm{C} 9$ & $-158.91(18)$ \\
\hline $\mathrm{C} 5-\mathrm{Fe} 1-\mathrm{C} 10-\mathrm{C} 9$ & $-76.36(17)$ \\
\hline $\mathrm{C} 3-\mathrm{Fe} 1-\mathrm{C} 10-\mathrm{C} 9$ & $-117.04(17)$ \\
\hline $\mathrm{C} 8-\mathrm{Fe} 1-\mathrm{C} 10-\mathrm{C} 9$ & $38.33(13)$ \\
\hline $\mathrm{C} 4-\mathrm{Fe} 1-\mathrm{C} 10-\mathrm{C} 9$ & $-45.3(4)$ \\
\hline $\mathrm{C} 7-\mathrm{Fe} 1-\mathrm{C} 10-\mathrm{C} 9$ & $82.13(15)$ \\
\hline $\mathrm{C} 6-\mathrm{Fe} 1-\mathrm{C} 10-\mathrm{C} 9$ & $119.4(2)$ \\
\hline $\mathrm{C} 1-\mathrm{Fe} 1-\mathrm{C} 10-\mathrm{C} 9$ & $169.3(3)$ \\
\hline $\mathrm{C} 6-\mathrm{C} 10-\mathrm{C} 9-\mathrm{C} 8$ & $0.4(3)$ \\
\hline $\mathrm{Fe} 1-\mathrm{C} 10-\mathrm{C} 9-\mathrm{C} 8$ & $-59.63(16)$ \\
\hline $\mathrm{C} 6-\mathrm{C} 10-\mathrm{C} 9-\mathrm{C} 16$ & $175.9(2)$ \\
\hline $\mathrm{Fe} 1-\mathrm{C} 10-\mathrm{C} 9-\mathrm{C} 16$ & $115.9(2)$ \\
\hline $\mathrm{C} 6-\mathrm{C} 10-\mathrm{C} 9-\mathrm{Fe} 1$ & $60.04(17)$ \\
\hline $\mathrm{C} 10-\mathrm{Fe} 1-\mathrm{C} 9-\mathrm{C} 8$ & $118.34(19)$ \\
\hline $\mathrm{C} 2-\mathrm{Fe} 1-\mathrm{C} 9-\mathrm{C} 8$ & $168.5(3)$ \\
\hline $\mathrm{C} 5-\mathrm{Fe} 1-\mathrm{C} 9-\mathrm{C} 8$ & $-118.57(15)$ \\
\hline $\mathrm{C} 3-\mathrm{Fe} 1-\mathrm{C} 9-\mathrm{C} 8$ & $-160.17(16)$ \\
\hline $\mathrm{C} 4-\mathrm{Fe} 1-\mathrm{C} 9-\mathrm{C} 8$ & $-77.80(17)$ \\
\hline $\mathrm{C} 7-\mathrm{Fe} 1-\mathrm{C} 9-\mathrm{C} 8$ & $37.66(14)$ \\
\hline $\mathrm{C} 6-\mathrm{Fe} 1-\mathrm{C} 9-\mathrm{C} 8$ & $81.07(15)$ \\
\hline $\mathrm{C} 1-\mathrm{Fe} 1-\mathrm{C} 9-\mathrm{C} 8$ & $-48.5(4)$ \\
\hline $\mathrm{C} 2-\mathrm{Fe} 1-\mathrm{C} 9-\mathrm{C} 10$ & $50.1(3)$ \\
\hline $\mathrm{C} 5-\mathrm{Fe} 1-\mathrm{C} 9-\mathrm{C} 10$ & $123.08(15)$ \\
\hline $\mathrm{C} 3-\mathrm{Fe} 1-\mathrm{C} 9-\mathrm{C} 10$ & $81.49(18)$ \\
\hline $\mathrm{C} 8-\mathrm{Fe} 1-\mathrm{C} 9-\mathrm{C} 10$ & $-118.34(19)$ \\
\hline $\mathrm{C} 4-\mathrm{Fe} 1-\mathrm{C} 9-\mathrm{C} 10$ & $163.86(15)$ \\
\hline $\mathrm{C} 7-\mathrm{Fe} 1-\mathrm{C} 9-\mathrm{C} 10$ & $-80.68(15)$ \\
\hline $\mathrm{C} 6-\mathrm{Fe} 1-\mathrm{C} 9-\mathrm{C} 10$ & $-37.27(14)$ \\
\hline $\mathrm{C} 1-\mathrm{Fe} 1-\mathrm{C} 9-\mathrm{C} 10$ & $-166.9(3)$ \\
\hline $\mathrm{C} 10-\mathrm{Fe} 1-\mathrm{C} 9-\mathrm{C} 16$ & $-115.7(2)$ \\
\hline $\mathrm{C} 2-\mathrm{Fe} 1-\mathrm{C} 9-\mathrm{C} 16$ & $-65.6(4)$ \\
\hline $\mathrm{C} 5-\mathrm{Fe} 1-\mathrm{C} 9-\mathrm{C} 16$ & $7.4(2)$ \\
\hline $\mathrm{C} 3-\mathrm{Fe} 1-\mathrm{C} 9-\mathrm{C} 16$ & $-34.2(2)$ \\
\hline $\mathrm{C} 8-\mathrm{Fe} 1-\mathrm{C} 9-\mathrm{C} 16$ & $125.9(3)$ \\
\hline $\mathrm{C} 4-\mathrm{Fe} 1-\mathrm{C} 9-\mathrm{C} 16$ & $48.1(2)$ \\
\hline $\mathrm{C} 7-\mathrm{Fe} 1-\mathrm{C} 9-\mathrm{C} 16$ & $163.6(2)$ \\
\hline $\mathrm{C} 6-\mathrm{Fe} 1-\mathrm{C} 9-\mathrm{C} 16$ & $-153.0(2)$ \\
\hline $\mathrm{C} 1-\mathrm{Fe} 1-\mathrm{C} 9-\mathrm{C} 16$ & $77.4(4)$ \\
\hline $\mathrm{N} 2-\mathrm{C} 15-\mathrm{C} 17-\mathrm{C} 16$ & $-1.7(3)$ \\
\hline $\mathrm{C} 14-\mathrm{C} 15-\mathrm{C} 17-\mathrm{C} 16$ & $179.2(2)$ \\
\hline $\mathrm{N} 2-\mathrm{C} 15-\mathrm{C} 17-\mathrm{C} 18$ & $177.4(2)$ \\
\hline $\mathrm{C} 14-\mathrm{C} 15-\mathrm{C} 17-\mathrm{C} 18$ & $-1.6(3)$ \\
\hline $\mathrm{C} 18-\mathrm{C} 17-\mathrm{C} 16-\mathrm{C} 9$ & $0.5(4)$ \\
\hline $\mathrm{C} 15-\mathrm{C} 17-\mathrm{C} 16-\mathrm{C} 9$ & $179.6(2)$ \\
\hline $\mathrm{C} 8-\mathrm{C} 9-\mathrm{C} 16-\mathrm{C} 17$ & $-8.6(4)$ \\
\hline
\end{tabular}

\begin{tabular}{|c|c|}
\hline $\mathrm{C} 12-\mathrm{C} 11-\mathrm{C} 13-\mathrm{C} 14$ & $0.0(4)$ \\
\hline $\mathrm{C} 15-\mathrm{C} 14-\mathrm{C} 13-\mathrm{C} 11$ & $0.4(4)$ \\
\hline $\mathrm{C} 3-\mathrm{C} 5-\mathrm{C} 4-\mathrm{C} 1$ & $0.1(3)$ \\
\hline $\mathrm{Fe} 1-\mathrm{C} 5-\mathrm{C} 4-\mathrm{C} 1$ & $59.75(18)$ \\
\hline $\mathrm{C} 3-\mathrm{C} 5-\mathrm{C} 4-\mathrm{Fe} 1$ & $-59.64(19)$ \\
\hline $\mathrm{C} 10-\mathrm{Fe} 1-\mathrm{C} 4-\mathrm{C} 5$ & $-41.3(4)$ \\
\hline $\mathrm{C} 2-\mathrm{Fe} 1-\mathrm{C} 4-\mathrm{C} 5$ & $80.8(2)$ \\
\hline $\mathrm{C} 9-\mathrm{Fe} 1-\mathrm{C} 4-\mathrm{C} 5$ & $-75.9(2)$ \\
\hline $\mathrm{C} 3-\mathrm{Fe} 1-\mathrm{C} 4-\mathrm{C} 5$ & $37.08(18)$ \\
\hline $\mathrm{C} 8-\mathrm{Fe} 1-\mathrm{C} 4-\mathrm{C} 5$ & $-118.34(17)$ \\
\hline $\mathrm{C} 7-\mathrm{Fe} 1-\mathrm{C} 4-\mathrm{C} 5$ & $-160.60(16)$ \\
\hline $\mathrm{C} 6-\mathrm{Fe} 1-\mathrm{C} 4-\mathrm{C} 5$ & $165.0(3)$ \\
\hline $\mathrm{C} 1-\mathrm{Fe} 1-\mathrm{C} 4-\mathrm{C} 5$ & $118.3(3)$ \\
\hline $\mathrm{C} 10-\mathrm{Fe} 1-\mathrm{C} 4-\mathrm{C} 1$ & $-159.7(3)$ \\
\hline $\mathrm{C} 2-\mathrm{Fe} 1-\mathrm{C} 4-\mathrm{C} 1$ & $-37.6(2)$ \\
\hline $\mathrm{C} 5-\mathrm{Fe} 1-\mathrm{C} 4-\mathrm{C} 1$ & $-118.3(3)$ \\
\hline $\mathrm{C} 9-\mathrm{Fe} 1-\mathrm{C} 4-\mathrm{C} 1$ & $165.8(2)$ \\
\hline $\mathrm{C} 3-\mathrm{Fe} 1-\mathrm{C} 4-\mathrm{C} 1$ & $-81.3(2)$ \\
\hline $\mathrm{C} 8-\mathrm{Fe} 1-\mathrm{C} 4-\mathrm{C} 1$ & $123.3(2)$ \\
\hline $\mathrm{C} 7-\mathrm{Fe} 1-\mathrm{C} 4-\mathrm{C} 1$ & $81.1(2)$ \\
\hline $\mathrm{C} 6-\mathrm{Fe} 1-\mathrm{C} 4-\mathrm{C} 1$ & $46.7(4)$ \\
\hline $\mathrm{C} 9-\mathrm{C} 10-\mathrm{C} 6-\mathrm{C} 7$ & $-0.4(3)$ \\
\hline $\mathrm{Fe} 1-\mathrm{C} 10-\mathrm{C} 6-\mathrm{C} 7$ & $58.99(17)$ \\
\hline $\mathrm{C} 9-\mathrm{C} 10-\mathrm{C} 6-\mathrm{Fe} 1$ & $-59.35(16)$ \\
\hline $\mathrm{C} 8-\mathrm{C} 7-\mathrm{C} 6-\mathrm{C} 10$ & $0.2(3)$ \\
\hline $\mathrm{Fe} 1-\mathrm{C} 7-\mathrm{C} 6-\mathrm{C} 10$ & $-58.51(17)$ \\
\hline $\mathrm{C} 8-\mathrm{C} 7-\mathrm{C} 6-\mathrm{Fe} 1$ & $58.70(17)$ \\
\hline $\mathrm{C} 2-\mathrm{Fe} 1-\mathrm{C} 6-\mathrm{C} 10$ & $-116.9(2)$ \\
\hline $\mathrm{C} 5-\mathrm{Fe} 1-\mathrm{C} 6-\mathrm{C} 10$ & $-43.6(4)$ \\
\hline $\mathrm{C} 9-\mathrm{Fe} 1-\mathrm{C} 6-\mathrm{C} 10$ & 38.14 (14) \\
\hline $\mathrm{C} 3-\mathrm{Fe} 1-\mathrm{C} 6-\mathrm{C} 10$ & $-75.4(2)$ \\
\hline $\mathrm{C} 8-\mathrm{Fe} 1-\mathrm{C} 6-\mathrm{C} 10$ & $82.44(15)$ \\
\hline $\mathrm{C} 4-\mathrm{Fe} 1-\mathrm{C} 6-\mathrm{C} 10$ & $167.2(3)$ \\
\hline $\mathrm{C} 7-\mathrm{Fe} 1-\mathrm{C} 6-\mathrm{C} 10$ & $119.7(2)$ \\
\hline $\mathrm{C} 1-\mathrm{Fe} 1-\mathrm{C} 6-\mathrm{C} 10$ & $-159.0(2)$ \\
\hline $\mathrm{C} 10-\mathrm{Fe} 1-\mathrm{C} 6-\mathrm{C} 7$ & $-119.7(2)$ \\
\hline $\mathrm{C} 2-\mathrm{Fe} 1-\mathrm{C} 6-\mathrm{C} 7$ & $123.4(2)$ \\
\hline $\mathrm{C} 5-\mathrm{Fe} 1-\mathrm{C} 6-\mathrm{C} 7$ & $-163.3(3)$ \\
\hline $\mathrm{C} 9-\mathrm{Fe} 1-\mathrm{C} 6-\mathrm{C} 7$ & $-81.56(16)$ \\
\hline $\mathrm{C} 3-\mathrm{Fe} 1-\mathrm{C} 6-\mathrm{C} 7$ & $164.90(18)$ \\
\hline $\mathrm{C} 8-\mathrm{Fe} 1-\mathrm{C} 6-\mathrm{C} 7$ & $-37.26(15)$ \\
\hline $\mathrm{C} 4-\mathrm{Fe} 1-\mathrm{C} 6-\mathrm{C} 7$ & $47.5(4)$ \\
\hline $\mathrm{C} 1-\mathrm{Fe} 1-\mathrm{C} 6-\mathrm{C} 7$ & $81.3(2)$ \\
\hline $\mathrm{C} 10-\mathrm{Fe} 1-\mathrm{C} 2-\mathrm{C} 1$ & $-161.20(17)$ \\
\hline $\mathrm{C} 5-\mathrm{Fe} 1-\mathrm{C} 2-\mathrm{C} 1$ & $81.0(2)$ \\
\hline $\mathrm{C} 9-\mathrm{Fe} 1-\mathrm{C} 2-\mathrm{C} 1$ & $162.4(2)$ \\
\hline $\mathrm{C} 3-\mathrm{Fe} 1-\mathrm{C} 2-\mathrm{C} 1$ & $118.7(3)$ \\
\hline $\mathrm{C} 8-\mathrm{Fe} 1-\mathrm{C} 2-\mathrm{C} 1$ & $-42.1(5)$ \\
\hline
\end{tabular}




\begin{tabular}{|c|c|c|c|}
\hline $\mathrm{C} 10-\mathrm{C} 9-\mathrm{C} 16-\mathrm{C} 17$ & $177.1(2)$ & $\mathrm{C} 4-\mathrm{Fe} 1-\mathrm{C} 2-\mathrm{C} 1$ & $37.53(18)$ \\
\hline $\mathrm{Fe} 1-\mathrm{C} 9-\mathrm{C} 16-\mathrm{C} 17$ & $-98.6(3)$ & $\mathrm{C} 7-\mathrm{Fe} 1-\mathrm{C} 2-\mathrm{C} 1$ & $-77.3(2)$ \\
\hline $\mathrm{C} 14-\mathrm{C} 15-\mathrm{N} 2-\mathrm{C} 12$ & $-0.1(4)$ & $\mathrm{C} 6-\mathrm{Fe} 1-\mathrm{C} 2-\mathrm{C} 1$ & $-118.69(19)$ \\
\hline $\mathrm{C} 17-\mathrm{C} 15-\mathrm{N} 2-\mathrm{C} 12$ & $-179.2(2)$ & $\mathrm{C} 10-\mathrm{Fe} 1-\mathrm{C} 2-\mathrm{C} 3$ & $80.1(2)$ \\
\hline $\mathrm{C} 10-\mathrm{C} 9-\mathrm{C} 8-\mathrm{C} 7$ & $-0.3(3)$ & $\mathrm{C} 5-\mathrm{Fe} 1-\mathrm{C} 2-\mathrm{C} 3$ & $-37.71(19)$ \\
\hline $\mathrm{C} 16-\mathrm{C} 9-\mathrm{C} 8-\mathrm{C} 7$ & $-175.3(2)$ & $\mathrm{C} 9-\mathrm{Fe} 1-\mathrm{C} 2-\mathrm{C} 3$ & $43.7(4)$ \\
\hline $\mathrm{Fe} 1-\mathrm{C} 9-\mathrm{C} 8-\mathrm{C} 7$ & $-59.58(17)$ & $\mathrm{C} 8-\mathrm{Fe} 1-\mathrm{C} 2-\mathrm{C} 3$ & $-160.8(3)$ \\
\hline $\mathrm{C} 10-\mathrm{C} 9-\mathrm{C} 8-\mathrm{Fe} 1$ & $59.29(15)$ & $\mathrm{C} 4-\mathrm{Fe} 1-\mathrm{C} 2-\mathrm{C} 3$ & $-81.2(2)$ \\
\hline $\mathrm{C} 16-\mathrm{C} 9-\mathrm{C} 8-\mathrm{Fe} 1$ & $-115.7(2)$ & $\mathrm{C} 7-\mathrm{Fe} 1-\mathrm{C} 2-\mathrm{C} 3$ & $164.06(18)$ \\
\hline $\mathrm{C} 10-\mathrm{Fe} 1-\mathrm{C} 8-\mathrm{C} 7$ & $80.51(16)$ & $\mathrm{C} 6-\mathrm{Fe} 1-\mathrm{C} 2-\mathrm{C} 3$ & $122.6(2)$ \\
\hline $\mathrm{C} 2-\mathrm{Fe} 1-\mathrm{C} 8-\mathrm{C} 7$ & $-46.3(4)$ & $\mathrm{C} 1-\mathrm{Fe} 1-\mathrm{C} 2-\mathrm{C} 3$ & $-118.7(3)$ \\
\hline $\mathrm{C} 5-\mathrm{Fe} 1-\mathrm{C} 8-\mathrm{C} 7$ & $-160.94(16)$ & $\mathrm{C} 15-\mathrm{N} 2-\mathrm{C} 12-\mathrm{C} 11$ & $0.6(5)$ \\
\hline $\mathrm{C} 9-\mathrm{Fe} 1-\mathrm{C} 8-\mathrm{C} 7$ & $119.1(2)$ & $\mathrm{C} 13-\mathrm{C} 11-\mathrm{C} 12-\mathrm{N} 2$ & $-0.6(5)$ \\
\hline $\mathrm{C} 3-\mathrm{Fe} 1-\mathrm{C} 8-\mathrm{C} 7$ & $166.0(3)$ & $\mathrm{C} 4-\mathrm{C} 5-\mathrm{C} 3-\mathrm{C} 2$ & $0.2(3)$ \\
\hline $\mathrm{C} 4-\mathrm{Fe} 1-\mathrm{C} 8-\mathrm{C} 7$ & $-119.28(16)$ & $\mathrm{Fe} 1-\mathrm{C} 5-\mathrm{C} 3-\mathrm{C} 2$ & $-59.7(2)$ \\
\hline $\mathrm{C} 6-\mathrm{Fe} 1-\mathrm{C} 8-\mathrm{C} 7$ & $36.99(15)$ & $\mathrm{C} 4-\mathrm{C} 5-\mathrm{C} 3-\mathrm{Fe} 1$ & $59.92(18)$ \\
\hline $\mathrm{C} 1-\mathrm{Fe} 1-\mathrm{C} 8-\mathrm{C} 7$ & $-78.15(19)$ & $\mathrm{C} 1-\mathrm{C} 2-\mathrm{C} 3-\mathrm{C} 5$ & $-0.4(3)$ \\
\hline $\mathrm{C} 10-\mathrm{Fe} 1-\mathrm{C} 8-\mathrm{C} 9$ & $-38.57(13)$ & $\mathrm{Fe} 1-\mathrm{C} 2-\mathrm{C} 3-\mathrm{C} 5$ & $59.86(19)$ \\
\hline $\mathrm{C} 2-\mathrm{Fe} 1-\mathrm{C} 8-\mathrm{C} 9$ & $-165.4(3)$ & $\mathrm{C} 1-\mathrm{C} 2-\mathrm{C} 3-\mathrm{Fe} 1$ & $-60.3(2)$ \\
\hline $\mathrm{C} 5-\mathrm{Fe} 1-\mathrm{C} 8-\mathrm{C} 9$ & $79.98(17)$ & $\mathrm{C} 10-\mathrm{Fe} 1-\mathrm{C} 3-\mathrm{C} 5$ & $123.48(17)$ \\
\hline $\mathrm{C} 3-\mathrm{Fe} 1-\mathrm{C} 8-\mathrm{C} 9$ & $46.9(3)$ & $\mathrm{C} 2-\mathrm{Fe} 1-\mathrm{C} 3-\mathrm{C} 5$ & $-118.6(3)$ \\
\hline $\mathrm{C} 4-\mathrm{Fe} 1-\mathrm{C} 8-\mathrm{C} 9$ & $121.64(15)$ & $\mathrm{C} 9-\mathrm{Fe} 1-\mathrm{C} 3-\mathrm{C} 5$ & $80.2(2)$ \\
\hline $\mathrm{C} 7-\mathrm{Fe} 1-\mathrm{C} 8-\mathrm{C} 9$ & $-119.1(2)$ & $\mathrm{C} 8-\mathrm{Fe} 1-\mathrm{C} 3-\mathrm{C} 5$ & $46.2(4)$ \\
\hline $\mathrm{C} 6-\mathrm{Fe} 1-\mathrm{C} 8-\mathrm{C} 9$ & $-82.09(15)$ & $\mathrm{C} 4-\mathrm{Fe} 1-\mathrm{C} 3-\mathrm{C} 5$ & $-37.06(17)$ \\
\hline $\mathrm{C} 1-\mathrm{Fe} 1-\mathrm{C} 8-\mathrm{C} 9$ & $162.77(15)$ & $\mathrm{C} 7-\mathrm{Fe} 1-\mathrm{C} 3-\mathrm{C} 5$ & $-163.4(3)$ \\
\hline $\mathrm{C} 9-\mathrm{C} 8-\mathrm{C} 7-\mathrm{C} 6$ & $0.1(3)$ & $\mathrm{C} 6-\mathrm{Fe} 1-\mathrm{C} 3-\mathrm{C} 5$ & $164.61(16)$ \\
\hline $\mathrm{Fe} 1-\mathrm{C} 8-\mathrm{C} 7-\mathrm{C} 6$ & $-59.03(18)$ & $\mathrm{C} 1-\mathrm{Fe} 1-\mathrm{C} 3-\mathrm{C} 5$ & $-80.8(2)$ \\
\hline $\mathrm{C} 9-\mathrm{C} 8-\mathrm{C} 7-\mathrm{Fe} 1$ & $59.10(16)$ & $\mathrm{C} 10-\mathrm{Fe} 1-\mathrm{C} 3-\mathrm{C} 2$ & $-117.9(2)$ \\
\hline $\mathrm{C} 10-\mathrm{Fe} 1-\mathrm{C} 7-\mathrm{C} 6$ & $37.37(15)$ & $\mathrm{C} 5-\mathrm{Fe} 1-\mathrm{C} 3-\mathrm{C} 2$ & $118.6(3)$ \\
\hline $\mathrm{C} 2-\mathrm{Fe} 1-\mathrm{C} 7-\mathrm{C} 6$ & $-76.1(2)$ & $\mathrm{C} 9-\mathrm{Fe} 1-\mathrm{C} 3-\mathrm{C} 2$ & $-161.2(2)$ \\
\hline $\mathrm{C} 5-\mathrm{Fe} 1-\mathrm{C} 7-\mathrm{C} 6$ & $166.1(3)$ & $\mathrm{C} 8-\mathrm{Fe} 1-\mathrm{C} 3-\mathrm{C} 2$ & $164.8(3)$ \\
\hline $\mathrm{C} 9-\mathrm{Fe} 1-\mathrm{C} 7-\mathrm{C} 6$ & $82.05(16)$ & $\mathrm{C} 4-\mathrm{Fe} 1-\mathrm{C} 3-\mathrm{C} 2$ & $81.6(2)$ \\
\hline $\mathrm{C} 3-\mathrm{Fe} 1-\mathrm{C} 7-\mathrm{C} 6$ & $-42.2(4)$ & $\mathrm{C} 7-\mathrm{Fe} 1-\mathrm{C} 3-\mathrm{C} 2$ & $-44.8(5)$ \\
\hline $\mathrm{C} 8-\mathrm{Fe} 1-\mathrm{C} 7-\mathrm{C} 6$ & $120.1(2)$ & $\mathrm{C} 6-\mathrm{Fe} 1-\mathrm{C} 3-\mathrm{C} 2$ & $-76.8(2)$ \\
\hline $\mathrm{C} 4-\mathrm{Fe} 1-\mathrm{C} 7-\mathrm{C} 6$ & $-160.39(15)$ & $\mathrm{C} 1-\mathrm{Fe} 1-\mathrm{C} 3-\mathrm{C} 2$ & $37.9(2)$ \\
\hline $\mathrm{C} 1-\mathrm{Fe} 1-\mathrm{C} 7-\mathrm{C} 6$ & $-117.96(19)$ & $\mathrm{C} 3-\mathrm{C} 2-\mathrm{C} 1-\mathrm{C} 4$ & $0.5(3)$ \\
\hline $\mathrm{C} 10-\mathrm{Fe} 1-\mathrm{C} 7-\mathrm{C} 8$ & $-82.78(16)$ & $\mathrm{Fe} 1-\mathrm{C} 2-\mathrm{C} 1-\mathrm{C} 4$ & $-59.6(2)$ \\
\hline $\mathrm{C} 2-\mathrm{Fe} 1-\mathrm{C} 7-\mathrm{C} 8$ & $163.7(2)$ & $\mathrm{C} 3-\mathrm{C} 2-\mathrm{C} 1-\mathrm{Fe} 1$ & $60.1(2)$ \\
\hline $\mathrm{C} 5-\mathrm{Fe} 1-\mathrm{C} 7-\mathrm{C} 8$ & $46.0(3)$ & $\mathrm{C} 5-\mathrm{C} 4-\mathrm{C} 1-\mathrm{C} 2$ & $-0.4(3)$ \\
\hline $\mathrm{C} 9-\mathrm{Fe} 1-\mathrm{C} 7-\mathrm{C} 8$ & $-38.10(15)$ & $\mathrm{Fe} 1-\mathrm{C} 4-\mathrm{C} 1-\mathrm{C} 2$ & $59.2(2)$ \\
\hline $\mathrm{C} 3-\mathrm{Fe} 1-\mathrm{C} 7-\mathrm{C} 8$ & $-162.4(4)$ & $\mathrm{C} 5-\mathrm{C} 4-\mathrm{C} 1-\mathrm{Fe} 1$ & $-59.61(19)$ \\
\hline $\mathrm{C} 4-\mathrm{Fe} 1-\mathrm{C} 7-\mathrm{C} 8$ & $79.47(18)$ & $\mathrm{C} 10-\mathrm{Fe} 1-\mathrm{C} 1-\mathrm{C} 2$ & $44.2(4)$ \\
\hline $\mathrm{C} 6-\mathrm{Fe} 1-\mathrm{C} 7-\mathrm{C} 8$ & $-120.1(2)$ & $\mathrm{C} 5-\mathrm{Fe} 1-\mathrm{C} 1-\mathrm{C} 2$ & $-81.4(2)$ \\
\hline $\mathrm{C} 1-\mathrm{Fe} 1-\mathrm{C} 7-\mathrm{C} 8$ & $121.90(19)$ & $\mathrm{C} 9-\mathrm{Fe} 1-\mathrm{C} 1-\mathrm{C} 2$ & $-158.1(3)$ \\
\hline $\mathrm{N} 2-\mathrm{C} 15-\mathrm{C} 14-\mathrm{C} 13$ & $-0.4(4)$ & $\mathrm{C} 3-\mathrm{Fe} 1-\mathrm{C} 1-\mathrm{C} 2$ & $-37.8(2)$ \\
\hline $\mathrm{C} 17-\mathrm{C} 15-\mathrm{C} 14-\mathrm{C} 13$ & $178.6(2)$ & $\mathrm{C} 8-\mathrm{Fe} 1-\mathrm{C} 1-\mathrm{C} 2$ & $165.02(19)$ \\
\hline $\mathrm{C} 10-\mathrm{Fe} 1-\mathrm{C} 5-\mathrm{C} 3$ & $-75.2(2)$ & $\mathrm{C} 4-\mathrm{Fe} 1-\mathrm{C} 1-\mathrm{C} 2$ & $-119.2(3)$ \\
\hline $\mathrm{C} 2-\mathrm{Fe} 1-\mathrm{C} 5-\mathrm{C} 3$ & $37.9(2)$ & $\mathrm{C} 7-\mathrm{Fe} 1-\mathrm{C} 1-\mathrm{C} 2$ & $122.7(2)$ \\
\hline
\end{tabular}




\section{supporting information}

$\begin{array}{llll}\mathrm{C} 9-\mathrm{Fe} 1-\mathrm{C} 5-\mathrm{C} 3 & -117.5(2) & \mathrm{C} 6-\mathrm{Fe} 1-\mathrm{C} 1-\mathrm{C} 2 & 80.1(2) \\ \mathrm{C} 8-\mathrm{Fe} 1-\mathrm{C} 5-\mathrm{C} 3 & -160.23(19) & \mathrm{C} 10-\mathrm{Fe} 1-\mathrm{C} 1-\mathrm{C} 4 & 163.4(3) \\ \mathrm{C} 4-\mathrm{Fe} 1-\mathrm{C} 5-\mathrm{C} 3 & 119.9(3) & \mathrm{C} 2-\mathrm{Fe} 1-\mathrm{C} 1-\mathrm{C} 4 & 119.2(3) \\ \mathrm{C} 7-\mathrm{Fe} 1-\mathrm{C} 5-\mathrm{C} 3 & 166.4(3) & \mathrm{C} 5-\mathrm{Fe} 1-\mathrm{C} 1-\mathrm{C} 4 & 37.87(18) \\ \mathrm{C} 6-\mathrm{Fe} 1-\mathrm{C} 5-\mathrm{C} 3 & -42.2(4) & \mathrm{C} 9-\mathrm{Fe} 1-\mathrm{C} 1-\mathrm{C} 4 & -38.8(5) \\ \mathrm{C} 1-\mathrm{Fe} 1-\mathrm{C} 5-\mathrm{C} 3 & 81.8(2) & \mathrm{C} 3-\mathrm{Fe} 1-\mathrm{C} 1-\mathrm{C} 4 & 81.4(2) \\ \mathrm{C} 10-\mathrm{Fe} 1-\mathrm{C} 5-\mathrm{C} 4 & 164.91(16) & \mathrm{C} 8-\mathrm{Fe} 1-\mathrm{C} 1-\mathrm{C} 4 & -75.8(2) \\ \mathrm{C} 2-\mathrm{Fe} 1-\mathrm{C} 5-\mathrm{C} 4 & -82.0(2) & \mathrm{C} 7-\mathrm{Fe} 1-\mathrm{C} 1-\mathrm{C} 4 & -118.11(19) \\ \mathrm{C} 9-\mathrm{Fe} 1-\mathrm{C} 5-\mathrm{C} 4 & 122.60(17) & \mathrm{C} 6-\mathrm{Fe} 1-\mathrm{C} 1-\mathrm{C} 4 & -160.66(17)\end{array}$

Hydrogen-bond geometry $\left(A,{ }^{\circ}\right)$

\begin{tabular}{lllll}
\hline$D-\mathrm{H} \cdots A$ & $D-\mathrm{H}$ & $\mathrm{H} \cdots A$ & $D \cdots A$ & $D-\mathrm{H} \cdots A$ \\
\hline $\mathrm{C} 16-\mathrm{H} 16 A \cdots \mathrm{N} 2$ & 0.93 & 2.41 & $2.804(3)$ & 105 \\
$\mathrm{C} 4-\mathrm{H} 4 A \cdots \mathrm{N} 1^{\mathrm{i}}$ & 0.98 & 2.62 & $3.538(4)$ & 156 \\
\hline
\end{tabular}

Symmetry code: (i) $-x+1,-y+2,-z$. 